\title{
Pushing the Annotation of Cellular Activities to a Higher Resolution: Predicting Functions at the Isoform Level
}

\author{
Wenyuan $\mathrm{Li}^{1 *}$, Chun-Chi Liu ${ }^{2} 4^{*}$, Shuli Kang ${ }^{1 *}$, Jian-Rong $\mathrm{Li}^{2}$ and Yu-Ting Tseng ${ }^{2}$, \\ and Xianghong Jasmine Zhou ${ }^{1} \xi$ \\ ${ }^{1}$ Molecular and Computational Biology Program, Department of Biological Sciences, University of \\ Southern California, Los Angeles, California 90089, USA \\ ${ }^{2}$ Institute of Genomics and Bioinformatics, National Chung Hsing University, Taiwan \\ ${ }^{3}$ Institute of Biomedical Sciences, National Chung Hsing University, Taiwan \\ ${ }^{4}$ Agricultural Biotechnology Center, National Chung Hsing University, Taiwan \\ * Joint first authors \\ $\xi$ To whom correspondence should be addressed. Tel: +1 213740 7055; Fax: +1 213740 2475; \\ Email:xjzhou@usc.edu
}

\begin{abstract}
In past decades, the experimental determination of protein functions was expensive and timeconsuming, so numerous computational methods were developed to speed up and guide the process. However, most of these methods predict protein functions at the gene level and do not consider the fact that protein isoforms (translated from alternatively spliced transcripts), not genes, are the actual function carriers. Now, high-throughput RNA-seq technology is providing unprecedented opportunities to unravel protein functions at the isoform level. In this article, we review recent progress in the high-resolution functional annotations of protein isoforms, focusing on two methods developed by the authors. Both methods can integrate multiple RNA-seq datasets for comprehensively characterizing functions of protein isoforms.
\end{abstract}

\section{Introduction}

Alternative splicing of pre-mRNAs is a key mechanism for increasing transcriptome and proteome complexity in eukaryotic cells. More than $90 \%$ of human multi-exon genes undergo alternative splicing $[1,2]$. The proteins translated from alternatively spliced mRNA have different amino acid sequences and structures, and can have distinct (even opposing) biological functions. Alternative splicing plays important roles in development, physiology, and a large number of human diseases including cancer [3-5]. It is also reported to be a driver of the evolution of phenotypic complexity in mammals $[1,6,7]$. Because of the limitations of current experimental techniques, there are very few functional annotations of protein isoforms available in the 
literature. Although recent years have seen an increase in the number of studies on isoformspecific functions, most functional annotations for proteins are still recorded at the gene level. Therefore, there is a great need for high-resolution functional annotations of proteins at the isoform level.

Over the past few decades, many computational methods have been developed to predict the functions of proteins $[8,9]$. These methods can leverage a large variety of genomic and proteomic data sources to achieve accurate functional inferences, but their input data are either gene-centric or biased to the canonical isoforms that are usually best studied in the literature. For example, protein-protein interaction data are generally recorded at the gene level, without including any information about which isoform was actually tested in the experiments. Even in cases where a specific transcript is annotated, it is usually the canonical isoform. Another example is protein sequence data, wherein (i) changing just a few amino acids can lead to distinct functions of protein isoforms of the same gene [10-13] and (ii) the canonical isoform's sequence is the most prevalent, the best documented, and often the longest. With such data, it is difficult to capture the divergence of isoform functions of the same gene. Therefore, the functions predicted by these methods mostly remain at the gene level [14-16].

Thanks to the advent of high-throughput RNA-seq technology, we can massively parallel sequence the whole set of transcript isoforms (translated into protein isoforms) at far higher resolution than ever before [17]. This technology provides unprecedented opportunities to systematically study protein functions at the isoform level. Many methods have been developed to efficiently and precisely quantify the expression levels of transcript isoforms [18-23] with less bias than prior data. With the rapid accumulation of high-quality RNA-seq datasets obtained under different conditions, we are more confident than ever in our ability to unravel the functions of protein isoforms. The expression values of isoforms from different RNA-seq datasets are not directly comparable, being easily biased by platforms and protocols. However, the correlations between expression profiles are comparable across datasets [24,25]. Therefore, we adopted coexpression networks as the data modeling and analysis paradigm [26] for protein isoforms. This framework has been successfully used to predict gene functions [27-30] and in many other integrative analyses [24-26,31-33], including functional module discovery.

This paper reviews two computational methods that we recently developed to systematically annotate the functions of protein isoforms [34,35]. Figure 1 illustrates the major features of the two methods. Both approaches are based on isoform co-expression networks derived from different RNA-seq studies or datasets.

The first method is a network-based, machine learning approach that integrates multiple isoform co-expression networks and propagates the functions of known genes to isoforms throughout the networks (Figure 1A) [34]. Because most existing functional annotations are assigned to genes, and each gene contains one or more isoforms, the known functions (or labels) are initially assigned to "bags" of nodes in the isoform networks. The learning algorithm then iteratively 


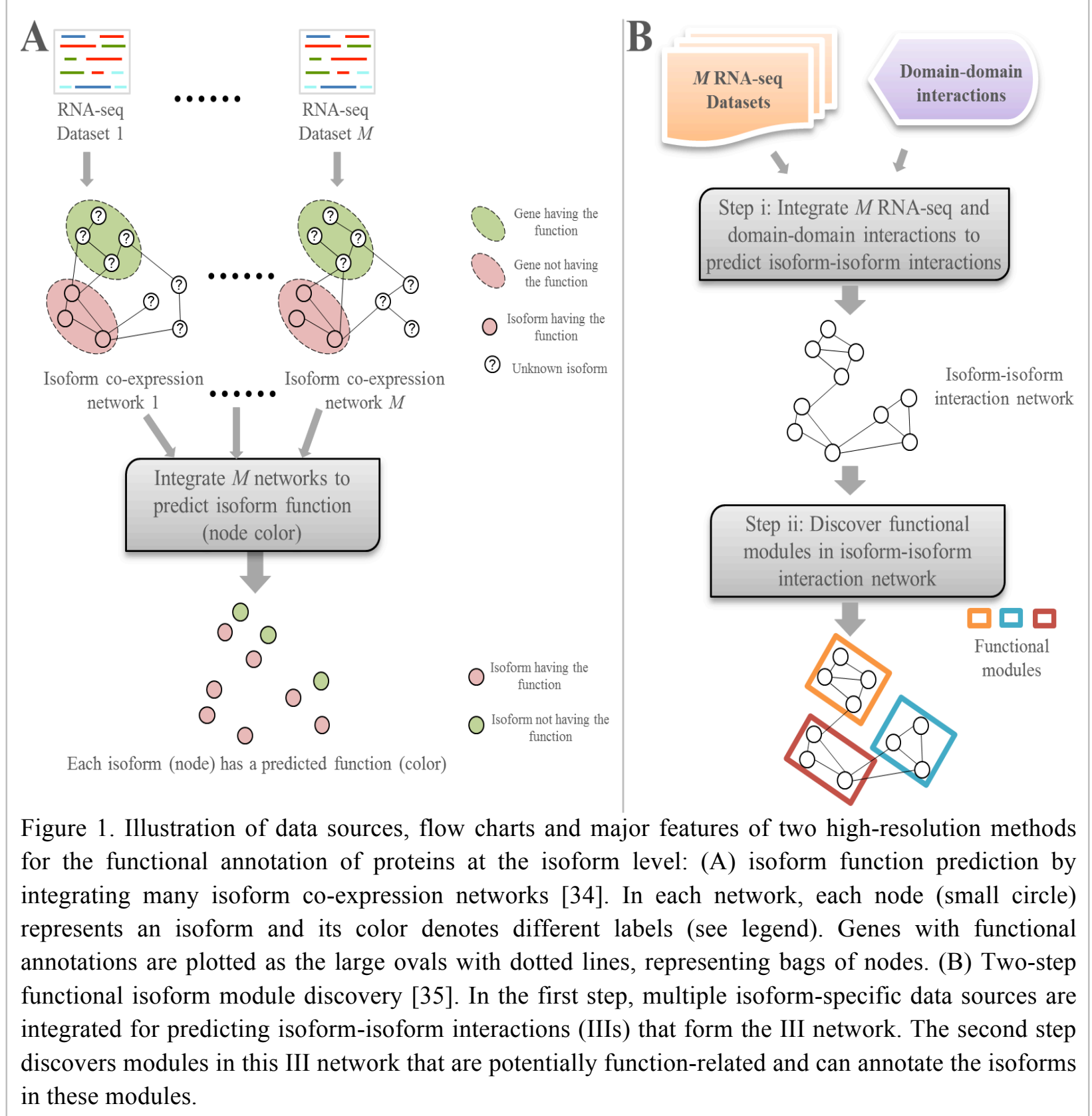

normalizes and updates labels in the network until convergence, and finally makes predictions for each isoform to be associated with a given function. As shown in Figure 1A, this method makes predictions for isoforms of unlabeled genes as well as identifying which isoforms of a labeled gene carry the function. This method obtained an AUC (area under the receiver operating characteristic curve) of 0.67 , and assigned 70,392 function annotations to15,572 isoforms. The results suggest that although many genes have isoforms carrying the same function, there is a substantial fraction of genes with functional variants. The results also show that functionally diverse isoforms usually have diverse expression patterns across tissues. 
The second approach is a two-step procedure (Figure 1B) [35]: (i) systematically predict genome-wide isoform-isoform interactions (IIIs) by integrating isoform co-expression networks and other data sources, then (ii) discover functional modules in the in silico III network. Recent studies $[36,37]$ indicate that isoform-level interactions are usually rewired by tissue-specific exons; thus, it is very likely that different isoforms of the same gene interact with different proteins and exert different functional roles. Comprehensively identifying protein-protein interactions at the isoform level (the first step) is important to systematically discover the cellular roles of protein isoforms, elucidate the exact composition of protein complexes, and gain insights into metabolic pathways. Since the function of an isoform can be inferred by its interacting partners in the III network, we can discover modules of densely interacting isoforms (the second step) that share similar functions. This method obtained 1,025 dense clusters of isoforms from the predicted III network, which are potential functional modules resolved at the isoform level.

\section{Isoform Function Prediction}

There are many network-based algorithms designed for integrating multiple gene co-expression networks to predict gene functions [27]. These algorithms use graph-based semi-supervised learning (GSSL), also known as the label propagation (LP) method [38-41]. This paradigm cannot be directly applied to isoform co-expression networks with functional labels at the gene level. As shown in Figure 1A, a "bag" of isoforms (nodes) of the same gene in the network is annotated by a single functional label and follows the rule (illustrated in Figure 1A) "If a gene is labeled as having a function, then we know that at least one of its isoforms has this function; on the other hand, if a gene is labeled as not having the function, then none of its isoforms has this function". This rule corresponds exactly to the definition of multiple-instance labeled data (or semi-labeled data), a type of annotated data where labels are assigned to groups of instances rather than individual instances. Therefore, existing LP methods are not suitable for our semilabeled data. Although there have been many multiple-instance learning algorithms in the literature $[42,43]$, none of them are applicable to our problem that has two unique features that do not exist in previous multiple-instance learning applications. Firstly, our isoform function prediction task is unconventional: we are more interested in knowing which isoforms "inherit" or "deviate from" the functions of their genes, than finding out whether some isoforms have new functions that are unknown for their host genes. Intuitively, we call the first type of prediction "inheritance prediction" and the second type "de novo prediction". Inheritance prediction can take full advantage of current gene function annotations, but is new to the literature. Most multiple-instance learning algorithms were designed for de novo prediction, and only a few are network-based learning [44,45]. Secondly, the task of integrating multiple semi-labeled isoform co-expression networks has never been done. Many studies in the area of gene function prediction have demonstrated that combining multiple data sources results in higher-quality function predictions [46]. We believe that the same principle is valid for isoform function prediction. 
Our method consists of two components as shown in Figure 2: (i) The network selection and combination component chooses the optimal subset of networks relevant to a given functional annotation among all input isoform co-expression networks, then aggregates them into a single

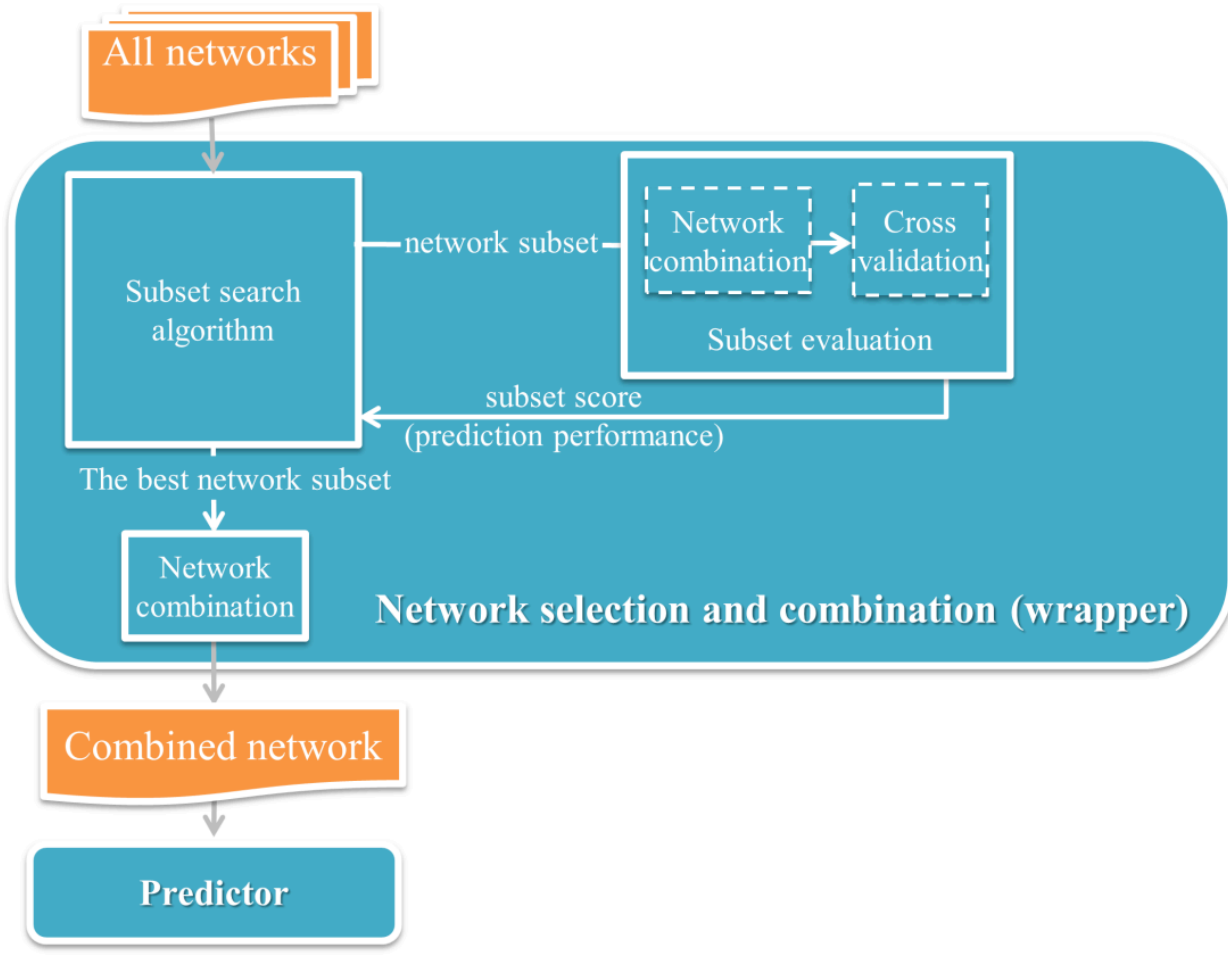

Figure 2. Flowchart of the proposed method with two components: "network selection and combination" and "predictor".

network. (ii) The predictor component is a novel label propagation method designed to work on the single semi-labeled isoform network. It takes the combined isoform co-expression network from the first component as its input, and returns function predictions for each isoform in the network.

\subsection{Method}

The predictor component takes a single multiple-instance (MI) labeled network as its input. Each bag of nodes (isoforms) in the network represents a single gene and has a label $+1,-1$, or 0 , indicating that this gene has, does not have or is unknown with respect to a given function. Following the MI labeling rule illustrated in Figure 1A, no node in positive bags is labeled, but all nodes in negative bags have negative labels (i.e., -1). This isoform network (with $N$ isoforms) is represented as an adjacency matrix $W=\left(w_{i j}\right)_{N \times N}$ where $w_{i j}$ denotes the intensity of association between isoforms $i$ and $j$. The normalized Laplacian of $W$ is defined as $L=D^{1 / 2} W D^{1 / 2}$, where $D$ is the diagonal matrix $D_{i i}=\Sigma_{j} w_{i j}$. We place all isoforms whose gene label is unknown (i.e. 0 ) into a single new category called the "unlabeled bag". Because these genes and their isoforms do not provide any constraints on the network, all their isoforms can be grouped without changing the 
result. We call this category a pseudo-bag because unlike the other bags, it contains isoforms from more than one gene. The predictor algorithm keeps track of and iteratively updates a realvalued prediction score $f_{i}[-1,1]$ for each node $i$, which is called a "soft label". Having now defined the network and terminology, the predictor algorithm is as follows:

Step 1. Initialize the soft label $f$ of each node (isoform) in a positive, negative, or unlabeled bag (gene) as $f=+1,-1$ or 0 , respectively.

Step 2. Clamp the soft labels of the nodes as follows:

a. For each node $i$ in the positive bags, $f_{i}^{\text {new }} \leftarrow f_{i}$ when $f_{i}>\varepsilon$ ( $\varepsilon$ is a positive number, close to zero), otherwise $f_{i}^{\text {new }} \leftarrow 0$. This is a "democratic" learning process that retains all qualified instances in the positive bag by demoting all nodes with negative soft labels (or even more strictly, nodes with $f<\varepsilon$ ) to zero. The threshold $\varepsilon$ should be inversely related to the number of instances $n$ in the positive bag. In practice, we used $\varepsilon=0.01 / \sqrt{n}$.

b. For each node $i$ in the negative bags, $f_{i}^{\text {new }} \leftarrow-1$. This step follows the MI labelling rule.

c. For each node $i$ in the unlabelled bag, the soft label $f$ remains unchanged: $f_{i}^{\text {new }} \leftarrow f_{i}$, because they serve as bridges for diffusing label information from the labeled bags to all nodes of the network.

d. Within each bag (whether positive, negative, or unlabeled), normalize the vector $f^{\text {new }}$ containing the scores of all nodes in the bag, so that their squared sum is $1: f$ $\leftarrow \operatorname{norm}\left(f^{n e w}\right)$. This step prevents the soft labels of the nodes from growing out of control after several iterations, and also guarantees that (a) the soft labels $f$ of nodes are proportional to their contributions to the bag, and (b) the larger the bag, the lower the $f$ scores of its nodes.

Step 3. Diffuse labels: $\boldsymbol{f} \leftarrow \mathrm{L} f$, where $\boldsymbol{f}$ is the vector of soft labels for all nodes. This step diffuses the label information of each node to its direct neighbors by a matrix multiplication.

Step 4. Repeat step 2 and 3 until $f$ converges.

After the soft labels $f$ of all nodes have converged, we need to make final predictions for the function of each node. For inheritance predictions, we assign a positive label to all nodes with non-zero $f$ scores in the positive bags. The criterion should be more stringent for de novo predictions. We empirically chose a threshold of 0.05 so that all nodes with $f$ no less than this threshold in the pseudo-bag are predicted to be positive. The algorithm is computationally efficient with a complexity of $\boldsymbol{O}(k|\mathrm{E}|+k|\mathrm{~V}|)$, where $k$ is the number of iterations (50 is usually enough for convergence), and $|\mathrm{E}|$ and $|\mathrm{V}|$ are the numbers of edges and nodes in the network respectively. The source code is available on our website (http://zhoulab.usc.edu/IsoFP). 

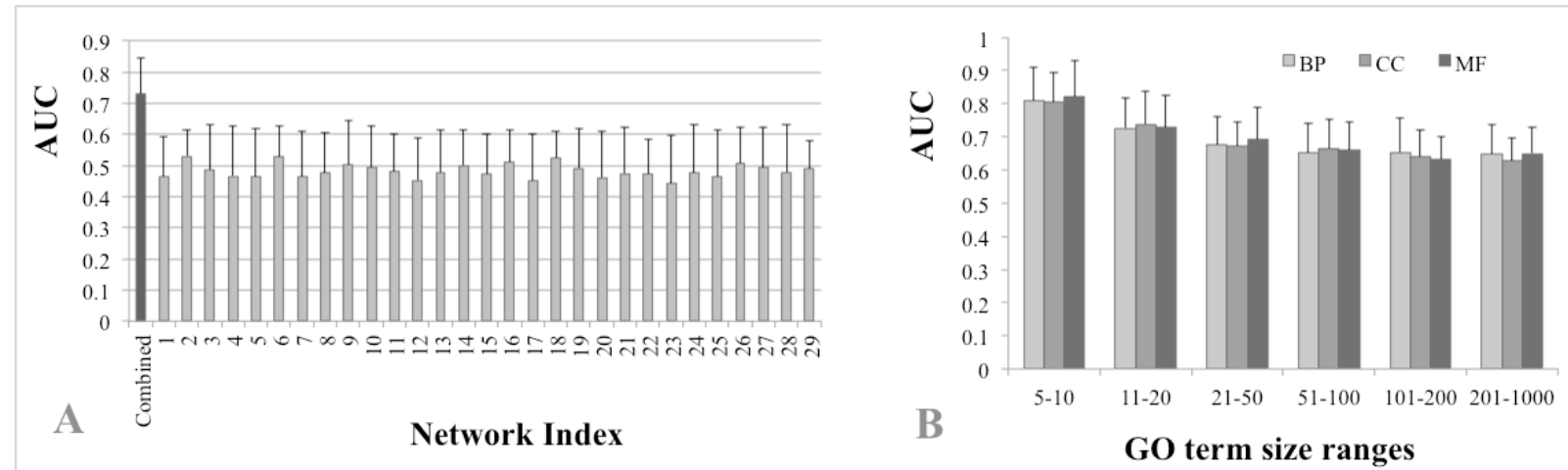

Figure 3. Prediction performance of the method. (A) Average AUC score across all GO terms, for each individual isoform co-expression network (blue bars), and for the optimally selected and combined subset of networks for each GO term (red bar). (B) Average AUC scores over all terms within one of three GO branches (BP - Biological Process, CC - Cellular Component, and MF - Molecular Function) and one of six ranges for the number of genes with the GO term ( 5 to 10,11 to 20,21 to 50,51 to 100,101 to 200 , or 201 to 1000). GO terms annotating fewer genes are more specific. The bars show average AUC scores (area under the receiver operating characteristic curve) for each group.

The network selection and combination component selects the most informative networks for each functional category among all input networks. It employs the wrapper method, a widely used feature selection strategy [47]. As shown in Figure 2, the wrapper method uses the prediction performance of network subsets to guide its search for the best subset. The performance score of a subset is obtained by applying the predictive model, with cross-validation, to the single network which combines all its member networks using equal weights. Our wrapper algorithm employs a greedy sequential forward strategy [48] to find the best subset. This strategy adds a new network to the current subset only if doing so improves the prediction performance.

\subsection{Results}

To construct the isoform co-expression networks, we selected 29 human RNA-seq datasets with at least 6 experiments from the Sequence Read Archive (SRA) database [49]. The datasets cover a wide range of experimental and physiological conditions. We ran the eXpress [19] with the Bowtie2 aligner [50] to obtain isoform expression values. NCBI Reference Sequences (RefSeq) mRNAs with protein sequences were used as transcriptome annotations, including 31,454 RefSeq IDs (Jan 2013 version) [51]. The mRNA level expression values were then converted directly into protein isoform expressions. In cases where two or more RefSeq mRNA sequences correspond to the same protein sequence, they are treated as belonging to a unique protein isoform, and their expression values are added. For each RNA-seq dataset, we constructed the isoform co-expression network by calculating the Pearson's correlation coefficient (PCC) between the expression profiles of each isoform pair and then normalizing the PCCs [24,52]. Gene Ontology (GO) data [53] and the UniProt Gene Ontology Annotation (UniProt-GOA) database [54] were used as the function categories and gene function annotations, respectively.

\subsubsection{Prediction performance}

We first applied our method to each single network. As shown in Figure 3A, no single network yielded an average AUC across all GO terms better than 0.53. The average AUC across all 29 
single networks is only 0.48 , even worse than a random guess (AUC 0.5). Therefore, we applied the wrapper method to selects an optimal subset of networks for each GO term, based on their "usefulness" to the specific prediction at hand. Note that different GO term may have different optimal subset of networks. Our wrapper method results in a dramatically increased AUC score of 0.67 , averaged across all GO terms (Figure $3 \mathrm{~A}$ ). This demonstrates the necessity of integrating multiple data sets for isoform function prediction.

GO annotations vary from highly specific functions that only involve a few genes to general categories such as "cell cycle" with many associated genes. To investigate whether the performance of our label propagation method is influenced by the number of positive bags in the network, we divided the GO terms into 24 groups, following a standard procedure used in previous gene function predictions [27], then evaluated the performance of our algorithm in each group. These groups are based on the major GO branches (BP, CC and MF) and on their sizes (the number of genes annotated with a GO term). Six size ranges were defined: [5,10], [11,20], [21,50], [51,100], [101,200], and [201,1000]). Figure 3B shows that the mean AUCs are higher than 0.6 in all GO groups. However, we also observed that GO terms with more associated genes have slightly worse prediction performance. This trend is consistent with previous gene function predictions [27]. A possible explanation is that genes associated with GO terms of larger size are usually more heterogeneous, thus it is harder to accurately predict the labels of their isoforms.

\subsubsection{Function annotations of human isoforms and literature validation}

We applied our method to 4,519 GO terms and generated functional predictions for the human protein isoforms. We obtained 70,392 isoform-level function predictions, 13,621 of which were de novo predictions, meaning that their host genes are not annotated positively or negatively with the predicted functions in the current GO database. In addition, we predicted the functions of 8,856 isoforms that have at least one annotation inherited from their host genes. To validate our predictions, we considered a number of apoptosis-regulating isoforms that are reported in the cancer-related literature, because the apoptosis process is usually distressed in cancer cells.

The human tumor suppressor gene TP53 is well-known for its role in inducing apoptosis and thus inhibiting tumorigenesis. According to its RefSeq annotation, $8 \mathrm{mRNA}$ isoforms are ascribed to TP53, corresponding to 7 unique protein products. The canonical isoform of TP53, p53a, is the full-length transcript composed of two transcription activation domains (TADs), a proline repeat domain (PXXP), a DNA binding domain (DBD), a nuclear localization signaling domain (NLS) and an oligomerization domain (OD). The other 6 protein products differ from the canonical form in TAD, PXXP, DBD and OD. Two of these, p53 $\beta$ and p53 $\gamma$, are C-terminal isoforms produced by partial intron retention. Along with p53 $\alpha$, they demonstrate positive regulation of the apoptotic pathway [55-57]. In $\mathrm{p} 53 \beta$ and $\mathrm{p} 53 \gamma$, the OD is replaced by a short peptide of 10 and 15 residues respectively. Isoform $\mathrm{p} 53 \beta$ is reported to enhance the transcriptional activity of $\mathrm{p} 53 \alpha$, and has weaker proapoptotic activity than $\mathrm{p} 53 \alpha[55,56]$. A recent study found that mutant $\mathrm{p} 53$ breast cancer patients with $\mathrm{p} 53 \gamma$ expressed have a particularly good prognosis compared to those without this isoform expressed [57], indicating the capacity of p53 $\gamma$ 
to induce apoptosis. Interestingly, two other isoforms, $\Delta 40 \mathrm{p} 53 \alpha$ and $\Delta 133 \mathrm{p} 53 \alpha$ are reported to have anti-apoptotic activity [55,58]. $\Delta 40$ p53 $\alpha$ lacks the first TAD, but the DBD and OD are intact. It is able to interact with $\mathrm{p} 53 \alpha$, negatively regulating its transcriptional activity via competitive binding to specific DNA regions. In this way, it behaves as a suppressor of the fulllength isoform $\mathrm{p} 53 \alpha$ [58]. $\Delta 133 \mathrm{p} 53 \alpha$ has even more amino acids deleted, losing both TADs and part of the OD. Thus, this isoform is an inhibitor of p53 $\alpha$ [55].

Table 1. Isoform function prediction results of four apoptotic genes

\begin{tabular}{|c|c|c|c|c|c|c|}
\hline \multirow{2}{*}{ Gene } & \multirow{2}{*}{ Isoform } & \multirow{2}{*}{$\begin{array}{c}\text { Predicted as a } \\
\text { regulator of } \\
\text { apoptosis? }\end{array}$} & \multicolumn{2}{|c|}{ Positive regulation? } & \multicolumn{2}{|c|}{ Negative regulation? } \\
\hline & & & Annotation & Prediction & Annotation & Prediction \\
\hline \multirow{5}{*}{ TP53 } & p53a & & & & $X$ & \\
\hline & p53 $\beta$ & & & & $X$ & $X$ \\
\hline & p53y & & & & $X$ & $x$ \\
\hline & $\Delta 40 p 53 \alpha$ & & $X$ & $X$ & & \\
\hline & $\Delta 133 p 53 \alpha$ & & $X$ & $X$ & & \\
\hline \multirow{2}{*}{ BCL2L1 } & Bcl-xL & & $X$ & $X$ & & \\
\hline & $\mathrm{Bcl}-\mathrm{xS}$ & & & $X$ & $X$ & $X$ \\
\hline \multirow{2}{*}{ CFLAR } & cFLIP-L & & & $\mathrm{X}$ & & $X$ \\
\hline & cFLIP-S & & $X$ & $X$ & & \\
\hline \multirow{2}{*}{ DNAJA3 } & Tid-1(L) & & & $X$ & $x$ & $x$ \\
\hline & $\operatorname{Tid}-1(\mathrm{~S})$ & & $X$ & $X$ & & \\
\hline
\end{tabular}

Note: Positive and negative results are represented by circles and crosses, respectively.

All five isoforms described above were correctly predicted by our method to have the GO term "regulation of apoptotic process" (GO:0042981) or any of its descendants (Table 1). We then asked whether the direction of the regulation could be also resolved at the isoform level. For each isoform, we checked our predictions for the two child terms "positive regulation of apoptotic process" (GO: 0043065) and "negative regulation of apoptotic process" (GO:0043066). It should be emphasized that since we performed predictions for each GO term independently, the same isoform can be assigned both positive regulation and negative regulation by different predictions. We therefore have ten predictions of regulation direction for the five isoforms. Surprisingly, even for TP53, a gene with many isoforms, our algorithm made the correct decision in nine out of ten predictions.

We also surveyed the literature on three other apoptosis regulatory genes (Table 1), all of which have both apoptosis-inducing and apoptosis-suppressing isoforms. BCL2L1has two isoforms: Bcl-xL inhibits and Bcl-xS promotes programmed cell death [59]. CFLAR, the CASP8 and FADD-like apoptosis regulator, has two well-annotated isoforms cFLIP-L and cFLIP-S. Both 
isoforms are inhibitors of apoptotic proteins [60], and cFLIP-L is also a promoter of apoptosis [61]. The third gene, DNAJA3, has two isoforms with opposite functions - one induces and the other represses the apoptotic process [62].

Our method successfully predicted the "regulation of apoptotic process" function for all 11 isoforms (including the five TP53 isoforms). Note that this excellent recall rate $(100 \%)$ cannot be attributed only to a preference for inheriting gene-level annotations in our predictions, because there is clearly a selective yet precise (only one false positive) inheritance from genes to isoforms on the child GO terms "positive regulation of apoptotic process" and "negative regulation of apoptotic process". This result suggests that our method can achieve both high recall and high precision. Indeed, on these four genes for which we could collect sufficient literature evidence, the overall accuracy of predictions on "positive regulation of apoptotic process" and "negative regulation of apoptotic process" are 72.7\% (8/11) and 81.8\% (9/11) respectively.

Furthermore, when we checked the predictions in detail, we found that BCL2L1 and CFLAR were not annotated with the GO term "positive regulation of apoptotic process" or any of its descendants in the input data. They were only annotated with the sibling term "negative regulation of apoptotic process", and thus were always treated as negative bags in our prediction. Our false negative predictions of these isoforms were therefore caused by misannotations in the GO database. This discovery indicates that our evaluation underestimates the real power of the method.

\section{Functional Isoform Module Discovery Using Isoform-Isoform Interaction Network}

Protein-protein interactions (PPIs) perform and regulate fundamental cellular processes. As a consequence, identifying interacting partners for a protein is essential to understand its functions. In recent years, remarkable progress has been made towards annotating all functional interactions between proteins in the cell. However, in both experimentally derived and computationally predicted protein-protein interactions, the term "protein" generally refers indiscriminately to "all isoforms of the respective gene." It is known that alternative splicing can modulate PPIs by altering protein structures and domain compositions, leading to the gain or loss of specific molecular interactions that could be key links of pathways [63]. It is very likely that different isoforms of the same protein interact with different proteins, thus exerting different functional roles. Therefore, in this work, we adopt a two-step functional annotation of protein isoforms (Figure 1B): first we identify protein-protein interactions at the isoform level, then we discover the functional isoform modules in these isoform-isoform interactions. 


\subsection{Prediction of isoform-isoform interactions}

In recent years, several studies have systematically predicted PPIs [64-68] and established PPI databases, e.g., OPHID [69], POINT [70], STRING [68] and PIPs [65]. With the exception of the IntAct database [71] which contains 116 human PPIs with isoform specifications, none of these databases contains isoform-level PPI data. In this work, we comprehensively predicted the possible interactions between isoforms by integrating a series of RNA-seq data and domaindomain interaction data. RNA-seq data provide two unique sources of information for reconstructing isoform-isoform interactions (IIIs): the absence or presence of specific isoforms under specific conditions, and the co-expression of two isoforms that may contribute to their interaction propensity. The resulting III network presents a higher-resolution map of PPIs, which is invaluable in characterizing biological processes and the specific cellular functions of isoforms.

We used the same RNA-seq data processing and isoform co-expression method detailed in Section 2.2. Among the 29 human RNA-seq datasets used in the first work, we selected the 19 datasets with at least 10 experiments for III prediction. We employed a logistic regression model to predict IIIs, because this model is suitable to describe the relationship between a binary response variable and a set of explanatory variables. The logistic regression is as follows:

$$
\operatorname{logit}\left(y_{i j}\right)=\alpha_{0}+\alpha_{1} E 1_{i j}+\alpha_{2} E 2_{i j}+\cdots+\alpha_{19} E 19_{i j}+\alpha_{20} D D I_{i j}
$$

where $\alpha_{0}, \alpha_{1}, \mathbf{K}, \alpha_{20}$ are regression coefficients and $y_{i j}$ is the probability of being an interaction between isoform $i$ and isoform $j$. The explanatory variables $D D I, E 1, E 2, \ldots, E 19$ are described as follows: (a) The $D D I_{i j}$ (domain-domain interaction) score: If the isoform pair ( $i$ and $j$ ) has a DDI in the DOMINE database [72,73], then we assign a DDI score to this pair based on the confidence level in the DOMINE database: 3 for a high-confidence prediction, 2 for a mediumconfidence prediction, and 1 for low-confidence prediction. If an isoform pair has multiple DDIs, we take the highest DDI score. (b) The variables, $E 1_{i j}, E 2_{i j}, \ldots, E 19_{i j}$, are the absolute values of Pearson's correlations of the isoform pair ( $i$ and $j$ ) derived from the 19 human RNA-seq datasets. Since the RNA-seq datasets vary in quality and data type, we rely on correlations rather than measures dependent on absolute expression values. The logistic regression model integrates all 19 human RNA-seq datasets and the coefficients of the variables $E 1_{i j}, E 2_{i j}, \ldots, E 19_{i j}$ reflect the quality of the RNA-seq datasets. 
For training and evaluating the predictive model, we need to construct a gold standard positive set (GSP) and a gold standard negative set (GSN) of IIIs. Currently, most PPI databases do not provide information at the level of isoforms, a significant challenge to constructing the GSP set. In the June 2013 version of the IntAct database [71], we identified 116 human PPIs with isoform specifications. In addition, we added those PPIs as IIIs to the GSP, if both of their interacting proteins have single isoforms. This increased the GSP set to 11,356 IIIs, covering 5,503 RefSeq IDs. We used the RefSeq IDs of the GSP set to construct the GSN set. The GSN contains isoform pairs in which one isoform is assigned to the plasma membrane cellular component, and

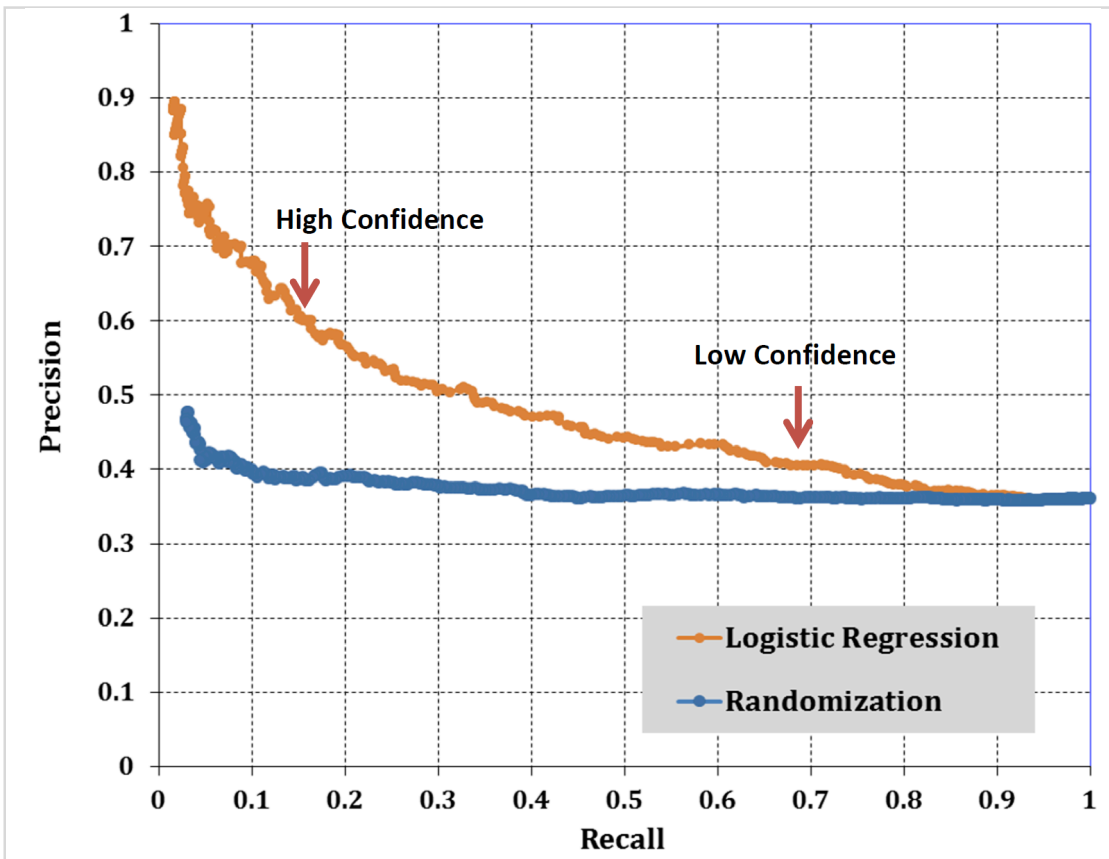

Figure 4. Precision and recall curve for the logistic regression model. At recall $15 \%$, the model achieves $60 \%$ precision (highconfidence prediction); at recall $68 \%$, the model achieves $40 \%$ precision (low-confidence prediction).

the other is assigned to the nuclear cellular component by the isoform-specific sub-cellular localization, in which we performed sequence-based predictions using CELLO [74]. To obtain the accurate isoform-specific annotation, we only used the cellular localization prediction results that consist of UniProt GO annotations.

The III prediction performance is shown in Figure 4. We have built a database, IIIDB, for accessing and managing predicted human IIIs. In the IIIDB, we provided two III prediction sets using the logistic regression model: (a) the high-confidence prediction, with logit score $>2.575$ (precision $60 \%$ and recall 15\%), resulted in $4,476 \mathrm{IIIs}$; and (b) the low-confidence prediction, with logit score $>1.692$ (precision $40 \%$ and recall 68\%). In addition, given a known PPI, the isoform pair with the best logit score is also selected as low-confidence prediction. Thus, each PPI has at least one isoform-isoform interaction. This resulted in 54,605 IIIs, which are used as the III network for isoform module discovery. 


\subsection{Functional Isoform module discovery}

To discover functional modules in the III network, we applied the MODES network clustering method [32] on the low-confidence III network to discover isoform modules with minimum module size 3, maximum module size 30, and density cutoff 0.7 . An important feature of MODES is that it can discover overlapping dense isoform modules, allowing one isoform to belong to multiple modules. We obtained 1,025 modules with an average size of 5.08 isoforms. To provide functional annotations and evaluate functional enrichment, we performed enrichment analyses with the GO [53] and KEGG pathway [75] databases. These databases contain proteinlevel annotations that provide approximate functional annotations for the isoforms.

To evaluate the significance, we randomly generated a set of modules with the same number and the same size distribution as the MODES isoform modules. As shown in Table 2, the MODES isoform modules discovered in the III network have a significantly higher functional enrichment rate than the random cases, showing the strong biological relevance of the predicted modules. The MODES isoform modules were used to build the isoform module database in the IIIDB, and all discovered isoform modules were listed in Additional file 1. We also stored the GO and KEGG enrichment results for all isoform modules in the IIIDB to provide potential functional annotations.

Table 2. The enrichment rate of isoform modules based on GO and pathway enrichments

\begin{tabular}{cccc}
\hline Isoform modules & $\begin{array}{c}\# \\
\text { madulec }\end{array}$ & $\begin{array}{c}\text { \% modules enriched with } \\
\mathrm{GO}^{\mathrm{a}}\end{array}$ & $\begin{array}{c}\text { \% modules enriched with } \\
\text { nathwav }\end{array}$ \\
\hline $\begin{array}{c}\text { MODES } \\
\text { mndillec }\end{array}$ & 1025 & $88.7 \%$ & $36.1 \%$ \\
Randomization & 1025 & $49.7 \%$ & $10.1 \%$ \\
\hline
\end{tabular}

${ }^{\mathrm{a}}$ The modules enriched with GO term (P-value $\left.<0.001\right)$.

${ }^{\mathrm{b}}$ The modules enriched with pathway $(P$-value $<0.01)$.

Although isoform-specific protein function studies are very rare, we found isoform-specific biological evidences with the isoform functions of the gene APP, which validated our MODES modules consisting of the isoforms of APP. APP is the amyloid beta precursor protein expressed in many tissues and concentrated in the neurons. APP has a strong association with Alzheimer's disease [76]. Among all MODES isoform modules, APP has 8 isoforms that are involved in 13 modules. The predominant isoforms of APP are APP695 (RefSeq NM_201414), APP751 (RefSeq NM_201413) and APP770 (RefSeq NM_000484) isoforms, in which APP695 is expressed in neuronal cells whereas APP751 and APP770 isoform are expressed in non-neuronal cells [76,77]. Table 3 lists all APP isoform modules and their enriched pathways: three APP695specific modules, three APP751-specific modules, two APP770-specific modules and three APP695-APP751-mixture modules. Interestingly, all of the APP695-specific modules enrich with Neurodegenerative/Alzheimer's diseases, whereas all of the APP751/APP770-specific modules do not have any enriched pathway, suggesting that the APP isoform specific modules are validated. 
Table 3. The APP isoform modules and their enriched pathways (modules are listed in the ascending order of their module indices)

\begin{tabular}{|c|c|c|}
\hline Module index & $\begin{array}{l}\text { Isoform members (denoted by the their host gene symbol and } \\
\text { their RefSeq ID) }\end{array}$ & Enriched Pathway \\
\hline Mod-20 & APP (NM_001136129), CNTN3 (NM_020872), APLP1 (NM_005166) & $\begin{array}{l}\text { hsa01510 Neurodegenerative } \\
\text { diseases }(P=8.34 \mathrm{E}-05)\end{array}$ \\
\hline Mod-21 & $\begin{array}{l}\text { APP (NM_201413, APP751), APBB3 (NM_006051), APBB3 } \\
\text { (NM_133174), APLP2 (NM_001142276) }\end{array}$ & \\
\hline Mod-170 & $\begin{array}{l}\text { APP (NM_000484, APP770), COL18A1 (NM_130445), TGM2 } \\
\text { (NM_004613) }\end{array}$ & \\
\hline Mod-201 & $\begin{array}{l}\text { APP (NM_201413, APP751), APP (NM_201414, APP695), APLP2 } \\
\text { (NM_001642), APBB1 (NM_145689), APLP1 (NM_005166), CNTN3 } \\
\text { (NM_020872) }\end{array}$ & $\begin{array}{l}\text { hsa05010 Alzheimer's disease }(P= \\
1.18 \mathrm{E}-04)\end{array}$ \\
\hline Mod-291 & $\begin{array}{l}\text { APP (NM_201413, APP751), APBB3 (NM_133174), APBB3 } \\
\text { (NM_133172), APLP2 (NM_001142276), APLP2 (NM_001243299) }\end{array}$ & \\
\hline Mod-292 & $\begin{array}{l}\text { APP (NM_201414, APP695), MAPT (NM_001123067), GSK3A } \\
\text { (NM_019884), MAPT (NM_001203251) }\end{array}$ & $\begin{array}{l}\text { hsa01510 Neurodegenerative } \\
\text { diseases }(P=8.34 \mathrm{E}-05)\end{array}$ \\
\hline Mod-417 & $\begin{array}{l}\text { APP (NM_001204303), APP (NM_001136131), GSK3A (NM_019884), } \\
\text { MAPT (NM_001203251) }\end{array}$ & $\begin{array}{l}\text { hsa01510 Neurodegenerative } \\
\text { diseases }(P=8.34 \mathrm{E}-05)\end{array}$ \\
\hline Mod-418 & $\begin{array}{l}\text { APP (NM_201414, APP695), APP (NM_201413, APP751), APBB3 } \\
\text { (NM_133172), APBB1 (NM_145689), APLP2 (NM_001142276), } \\
\text { APLP2 (NM_001642) }\end{array}$ & $\begin{array}{l}\text { hsa01510 Neurodegenerative } \\
\text { diseases }(P=8.34 \mathrm{E}-05)\end{array}$ \\
\hline Mod-446 & $\begin{array}{l}\text { APP (NM_201414, APP695), PSEN1 (NM_000021), PRAM1 } \\
\text { (NM_032152), APBA1 (NM_001163) }\end{array}$ & $\begin{array}{l}\text { hsa01510 Neurodegenerative } \\
\text { diseases }(P=3.95 E-05)\end{array}$ \\
\hline Mod-547 & $\begin{array}{l}\text { APP (NM_201414, APP695), APP (NM_201413, APP751), APLP1 } \\
\text { (NM_005166), APLP2 (NM_001642), APBB3 (NM_133173) }\end{array}$ & $\begin{array}{l}\text { hsa01510 Neurodegenerative } \\
\text { diseases }(P=8.34 \mathrm{E}-05)\end{array}$ \\
\hline Mod-601 & $\begin{array}{l}\text { APP (NM_201414, APP695), APBB1 (NM_001257325), APLP2 } \\
\text { (NM_001642), APLP2 (NM_001142278) }\end{array}$ & $\begin{array}{l}\text { hsa05010 Alzheimer's disease }(P= \\
3.95 E-05)\end{array}$ \\
\hline Mod-602 & $\begin{array}{l}\text { APP (NM_201413, APP751), APP (NM_001204302), APLP2 } \\
\text { (NM_001142276), APBB3 (NM_133174), APLP2 (NM_001243299), } \\
\text { APLP2 (NM_001642) }\end{array}$ & \\
\hline Mod-654 & $\begin{array}{l}\text { APP (NM_000484, APP770), APP (NM_001136130), APLP2 } \\
\text { (NM_001142277), APBB2 (NM_001166050), APLP2 (NM_001642), }\end{array}$ & \\
\hline
\end{tabular}

\section{Discussion and Conclusion}

Although alternative transcript processing is a ubiquitous mechanism for generating functional diversity in genes, little is known about the precise functions of individual isoforms and their translated proteins. Owing to the limitations of experimental techniques, most large-scale genomic data are profiled at the gene level but not at the isoform level. Thus most existing function prediction methods were designed for integrating these gene-level data sources for gene function predictions, and cannot move to the isoform level. Now, RNA-seq techniques can profile isoform expressions, opening the door to systematic protein function predictions at the isoform level. Unlike gene function prediction, isoform function prediction faces a unique challenge: a serious dearth of training data, because most known functional annotations are at the 
gene level. Therefore, in this review, we summarized recent progress in this direction by the authors. The first method models the gene-isoform relationships as multiple-instance labeled data and develops a novel multiple instance learning algorithm to predict isoform functions based on known gene function annotations. The second method circumvents this problem by exploring the predicted isoform-isoform interaction network to discover modules in which densely interconnected isoforms potentially have similar functions. However, two issues need to be discussed.

\subsection{Pipeline of estimating isoform expressions from RNA-seq data}

We estimated isoform expression levels from RNA-seq data. Since this study uses a large number of RNA-seq datasets were used, it is important to choose the pipeline with the right trade-off between computation speed and estimation quality. Several transcript quantification tools exist, such as Cufflinks[18], eXpress[19], RSEM[20], and SLIDE[21]. We compared two most popular isoform abundance estimation pipelines, tophat + cufflinks and bowtie $2+e X p r e s s$, in terms of runtime and reproducibility. Both pipelines generated reproducible isoform abundances in two replicates of three ENCODE human cell types[78]. However, bowtie2+eXpress runs much faster than tophat+cufflinks, because bowtie $2+$ eXpress works directly on the transcript sequences, without introducing genomic information. In addition, eXpress provides an online streaming mechanism, so that bowtie 2 and eXpress can run at the same time using the pipe mechanism of Unix. Also, since the function annotations were resolved at the protein level, it is not necessary to distinguish mRNAs that differ in the untranslated regions. Therefore we reduced the computational cost using only the protein-coding sequence of each RefSeq mRNA in the expression evaluation.

\subsection{Performance evaluation}

Due to the lack of curated isoform function data, we used the annotations of single-isoform genes to assess the prediction performance of two approaches. However, multiple isoforms of the same gene may share the same promoter regions and some consensus exons, which are not generally shared by a given pair of single-isoform genes. That is, the relationships between single-isoform genes and those between multiple isoforms of the same genes are different. Therefore, both methods based on the single-isoform genes may not be best choice for function and interaction predictions of those isoforms whose host genes have multiple isoforms. This issue will eventually be addressed by training our model on functional annotations and interactions at isoform-level resolution when they become available in the future.

In future studies, as more and more RNA-seq datasets become available, these two integrative methods will produce more accurate functional annotations of isoforms and their predictions can be validated by more experimental evidence of specific isoform functions. 


\section{Acknowledgement}

This work was supported by the National Institutes of Health Grants NHLBI MAPGEN U01HL108634 and NIGMS R01GM105431 (to X.J.Z.), the National Science Foundation grant 0747475 (to X.J.Z.), and the Taiwan Ministry of Science and Technology grant MOST 1032320-B-005-004 (to C.C.L.).

\section{References}

[1] E.T. Wang, R. Sandberg, S. Luo, I. Khrebtukova, L. Zhang, C. Mayr, et al., Alternative isoform regulation in human tissue transcriptomes., Nature. 456 (2008) 470-6. doi:10.1038/nature07509.

[2] Q. Pan, O. Shai, L.J. Lee, B.J. Frey, B.J. Blencowe, Deep surveying of alternative splicing complexity in the human transcriptome by high-throughput sequencing., Nat. Genet. 40 (2008) 1413-5. doi:10.1038/ng.259.

[3] A.J. Matlin, F. Clark, C.W.J. Smith, Understanding alternative splicing: towards a cellular code, Nat Rev Mol Cell Biol. 6 (2005) 386-398.

[4] J.-Y. Tang, J.-C. Lee, M.-F. Hou, C.-L. Wang, C.-C. Chen, H.-W. Huang, et al., Alternative splicing for diseases, cancers, drugs, and databases., ScientificWorldJournal. 2013 (2013) 703568. doi: $10.1155 / 2013 / 703568$.

[5] M.G. Poulos, R. Batra, K. Charizanis, M.S. Swanson, Developments in RNA splicing and disease., Cold Spring Harb. Perspect. Biol. 3 (2011) a000778. doi:10.1101/cshperspect.a000778.

[6] E.S. Lander, L.M. Linton, B. Birren, C. Nusbaum, M.C. Zody, J. Baldwin, et al., Initial sequencing and analysis of the human genome., Nature. 409 (2001) 860-921. doi: $10.1038 / 35057062$.

[7] J.M. Johnson, J. Castle, P. Garrett-Engele, Z. Kan, P.M. Loerch, C.D. Armour, et al., Genomewide survey of human alternative pre-mRNA splicing with exon junction microarrays., Science. 302 (2003) 2141-4. doi:10.1126/science.1090100.

[8] R. Sharan, I. Ulitsky, R. Shamir, Network-based prediction of protein function., Mol. Syst. Biol. 3 (2007) 88. doi:10.1038/msb4100129.

[9] G. Pandey, V. Kumar, M. Steinbach, C.L. Meyers, Computational Approaches to Protein Function Prediction, Wiley-Interscience, New York, NY, 2012.

[10] K. Vogan, D. Underhill, P. Gros, An alternative splicing event in the Pax-3 paired domain identifies the linker region as a key determinant of paired domain DNA-binding activity, Mol. Cell. Biol. 16 (1996) 6677-6686.

[11] S.A.K. Merediz, Cloning of an interferon regulatory factor 2 isoform with different regulatory ability, Nucleic Acids Res. 28 (2000) 4219-4224. 
[12] C. -a. A. Hu, Molecular Enzymology of Mammalian Delta 1-Pyrroline-5-carboxylate Synthase. ALTERNATIVE SPLICE DONOR UTILIZATION GENERATES ISOFORMS WITH DIFFERENT SENSITIVITY TO ORNITHINE INHIBITION, J. Biol. Chem. 274 (1999) 67546762.

[13] M. Yan, L.C. Wang, S.G. Hymowitz, S. Schilbach, J. Lee, A. Goddard, et al., Two-amino acid molecular switch in an epithelial morphogen that regulates binding to two distinct receptors., Science. 290 (2000) 523-7.

[14] J. Gillis, P. Pavlidis, Characterizing the state of the art in the computational assignment of gene function: lessons from the first critical assessment of functional annotation (CAFA)., BMC Bioinformatics. 14 Suppl 3 (2013) S15.

[15] T.M. Murali, C.-J. Wu, S. Kasif, The art of gene function prediction., Nat. Biotechnol. 24 (2006) 1474-5; author reply 1475-6. doi:10.1038/nbt1206-1474.

[16] P. Pavlidis, J. Gillis, Progress and challenges in the computational prediction of gene function using networks., F1000Research. 1 (2012) 14. doi:10.12688/f1000research.1-14.v1.

[17] U. Nagalakshmi, K. Waern, M. Snyder, RNA-Seq: a method for comprehensive transcriptome analysis., Curr. Protoc. Mol. Biol. Chapter 4 (2010) Unit 4.11.1-13. doi:10.1002/0471142727.mb0411s89.

[18] C. Trapnell, B.A. Williams, G. Pertea, A. Mortazavi, G. Kwan, M.J. van Baren, et al., Transcript assembly and quantification by RNA-Seq reveals unannotated transcripts and isoform switching during cell differentiation., Nat. Biotechnol. 28 (2010) 511-5.

[19] A. Roberts, L. Pachter, Streaming fragment assignment for real-time analysis of sequencing experiments., Nat. Methods. 10 (2013) 71-3.

[20] B. Li, C.N. Dewey, RSEM: accurate transcript quantification from RNA-Seq data with or without a reference genome., BMC Bioinformatics. 12 (2011) 323.

[21] J.J. Li, C.-R. Jiang, J.B. Brown, H. Huang, P.J. Bickel, Sparse linear modeling of next-generation mRNA sequencing (RNA-Seq) data for isoform discovery and abundance estimation., Proc. Natl. Acad. Sci. U. S. A. 108 (2011) 19867-72. doi:10.1073/pnas.1113972108.

[22] T. Steijger, J.F. Abril, P.G. Engström, F. Kokocinski, M. Akerman, T. Alioto, et al., Assessment of transcript reconstruction methods for RNA-seq., Nat. Methods. 10 (2013) 1177-84. doi:10.1038/nmeth.2714.

[23] R. Patro, S.M. Mount, C. Kingsford, Sailfish enables alignment-free isoform quantification from RNA-seq reads using lightweight algorithms., Nat. Biotechnol. 32 (2014) 462-4. doi:10.1038/nbt.2862.

[24] W. Li, C.-C. Liu, T. Zhang, H. Li, M.S. Waterman, X.J. Zhou, Integrative analysis of many weighted co-expression networks using tensor computation., PLoS Comput. Biol. 7 (2011) e1001106. 
[25] W. Li, H. Hu, Y. Huang, H. Li, M.R. Mehan, J. Nunez-Iglesias, et al., Frequent Pattern Discovery in Multiple Biological Networks: Patterns and Algorithms, Stat. Biosci. 4 (2011) 157-176. doi:10.1007/s12561-011-9047-0.

[26] M. Rotival, E. Petretto, Leveraging gene co-expression networks to pinpoint the regulation of complex traits and disease, with a focus on cardiovascular traits., Brief. Funct. Genomics. 13 (2014) 66-78. doi:10.1093/bfgp/elt030.

[27] S. Mostafavi, D. Ray, D. Warde-Farley, C. Grouios, Q. Morris, GeneMANIA: a real-time multiple association network integration algorithm for predicting gene function., Genome Biol. 9 Suppl 1 (2008) S4.

[28] Y.-H. Liang, B. Cai, F. Chen, G. Wang, M. Wang, Y. Zhong, et al., Construction and validation of a gene co-expression network in grapevine (Vitis vinifera. L.), Hortic. Res. 1 (2014) 14040. doi:10.1038/hortres.2014.40.

[29] F. Luo, Y. Yang, J. Zhong, H. Gao, L. Khan, D.K. Thompson, et al., Constructing gene coexpression networks and predicting functions of unknown genes by random matrix theory., BMC Bioinformatics. 8 (2007) 299. doi:10.1186/1471-2105-8-299.

[30] D.C.J. Wong, C. Sweetman, C.M. Ford, Annotation of gene function in citrus using gene expression information and co-expression networks., BMC Plant Biol. 14 (2014) 186. doi:10.1186/1471-2229-14-186.

[31] W. Li, C. Dai, C.-C. Liu, X.J. Zhou, Algorithm to identify frequent coupled modules from twolayered network series: application to study transcription and splicing coupling., J. Comput. Biol. 19 (2012) 710-30. doi:10.1089/cmb.2012.0025.

[32] H. Hu, X. Yan, Y. Huang, J. Han, X.J. Zhou, Mining Coherent Dense Subgraphs across Massive Biological Networks for Functional Discovery, Bioinformatics. 21 (2005) i213-221.

[33] X. Xiao, A. Moreno-Moral, M. Rotival, L. Bottolo, E. Petretto, Multi-tissue analysis of coexpression networks by higher-order generalized singular value decomposition identifies functionally coherent transcriptional modules., PLoS Genet. 10 (2014) e1004006. doi:10.1371/journal.pgen.1004006.

[34] W. Li, S. Kang, C.-C. Liu, S. Zhang, Y. Shi, Y. Liu, et al., High-resolution functional annotation of human transcriptome: predicting isoform functions by a novel multiple instance-based label propagation method., Nucleic Acids Res. (2013). doi:10.1093/nar/gkt1362.

[35] Y.-T. Tseng, W. Li, C.-H. Chen, S. Zhang, J.J. Chen, X. Zhou, et al., IIIDB: a database for isoform-isoform interactions and isoform network modules., BMC Genomics. 16 Suppl 2 (2015) S10. doi:10.1186/1471-2164-16-S2-S10.

[36] M. Buljan, G. Chalancon, S. Eustermann, G.P. Wagner, M. Fuxreiter, A. Bateman, et al., TissueSpecific Splicing of Disordered Segments that Embed Binding Motifs Rewires Protein Interaction Networks., Mol. Cell. 46 (2012) 871-83. doi:10.1016/j.molcel.2012.05.039. 
[37] J.D. Ellis, M. Barrios-Rodiles, R. Colak, M. Irimia, T. Kim, J.A. Calarco, et al., Tissue-specific alternative splicing remodels protein-protein interaction networks., Mol. Cell. 46 (2012) 884-92. doi:10.1016/j.molcel.2012.05.037.

[38] X. Zhu, Z. Ghahramani, Learning from labeled and unlabeled data with label propagation, 2002.

[39] Y. Fujiwara, G. Irie, Efficient Label Propagation, in: Proc. 31st Int. Conf. Mach. Learn., 2014: pp. 784-792.

[40] T. Kato, H. Kashima, M. Sugiyama, Robust Label Propagation on Multiple Networks, IEEE Trans. Neural Networks. 20 (2009) 35-44.

[41] W. Liu, J. Wang, S.-F. Chang, Robust and Scalable Graph-Based Semisupervised Learning, Proc. IEEE. 100 (2012) 2624-2638.

[42] S. Andrews, I. Tsochantaridis, T. Hofmann, Support vector machines for multiple-instance learning, in: Proc. Adv. Neural Inf. Process. Syst., 2002: pp. 561-568.

[43] J. Amores, Multiple instance classification: Review, taxonomy and comparative study, Artif. Intell. 201 (2013) 81-105. doi:10.1016/j.artint.2013.06.003.

[44] Y. Jia, C. Zhang, Instance-level semisupervised multiple instance learning, in: Proc. 23rd Natl. Conf. Artif. Intell., AAAI Press, 2008: pp. 640-645.

[45] C. Wang, L. Zhang, H.-J. Zhang, Graph-based multiple-instance learning for object-based image retrieval, in: Proceeding 1st ACM Int. Conf. Multimed. Inf. Retr. - MIR '08, ACM Press, New York, New York, USA, 2008: pp. 156-163.

[46] W. Noble, A. Ben-Hur, Integrating information for protein function prediction, in: BioinformaticsFrom Genomes to Ther., 2007: pp. 1297-1314.

[47] Y. Saeys, I. Inza, P. Larrañaga, A review of feature selection techniques in bioinformatics., Bioinformatics. 23 (2007) 2507-17.

[48] A model-free greedy gene selection for microarray sample class prediction, (n.d.).

[49] R. Leinonen, H. Sugawara, M. Shumway, The sequence read archive., Nucleic Acids Res. 39 (2011) D19-21.

[50] Y. Liu, B. Schmidt, Long read alignment based on maximal exact match seeds., Bioinformatics. 28 (2012) i318-i324.

[51] K.D. Pruitt, T. Tatusova, G.R. Brown, D.R. Maglott, NCBI Reference Sequences (RefSeq): current status, new features and genome annotation policy., Nucleic Acids Res. 40 (2012) D130-5.

[52] M. Xu, M.-C.J. Kao, J. Nunez-Iglesias, J.R. Nevins, M. West, X.J. Zhou, An integrative approach to characterize disease-specific pathways and their coordination: a case study in cancer., BMC Genomics. 9 Suppl 1 (2008) S12. doi:10.1186/1471-2164-9-S1-S12. 
[53] M. Ashburner, C.A. Ball, J.A. Blake, D. Botstein, H. Butler, J.M. Cherry, et al., Gene ontology: tool for the unification of biology. The Gene Ontology Consortium., Nat. Genet. 25 (2000) 25-9. doi: $10.1038 / 75556$.

[54] D. Barrell, E. Dimmer, R.P. Huntley, D. Binns, C. O'Donovan, R. Apweiler, The GOA database in 2009--an integrated Gene Ontology Annotation resource., Nucleic Acids Res. 37 (2009) D396403.

[55] J.-C. Bourdon, K. Fernandes, F. Murray-Zmijewski, G. Liu, A. Diot, D.P. Xirodimas, et al., p53 isoforms can regulate p53 transcriptional activity., Genes Dev. 19 (2005) 2122-37.

[56] K. Fujita, A.M. Mondal, I. Horikawa, G.H. Nguyen, K. Kumamoto, J.J. Sohn, et al., p53 isoforms Delta133p53 and p53beta are endogenous regulators of replicative cellular senescence., Nat. Cell Biol. 11 (2009) 1135-42.

[57] J.-C. Bourdon, M.P. Khoury, A. Diot, L. Baker, K. Fernandes, M. Aoubala, et al., p53 mutant breast cancer patients expressing p53 $\gamma$ have as good a prognosis as wild-type p53 breast cancer patients., Breast Cancer Res. 13 (2011) R7.

[58] S. Courtois, G. Verhaegh, S. North, M.-G. Luciani, P. Lassus, U. Hibner, et al., DeltaN-p53, a natural isoform of $\mathrm{p} 53$ lacking the first transactivation domain, counteracts growth suppression by wild-type p53., Oncogene. 21 (2002) 6722-8.

[59] L.H. Boise, M. González-García, C.E. Postema, L. Ding, T. Lindsten, L.A. Turka, et al., bcl-x, a bcl-2-related gene that functions as a dominant regulator of apoptotic cell death., Cell. 74 (1993) 597-608.

[60] A. Krueger, I. Schmitz, S. Baumann, P.H. Krammer, S. Kirchhoff, Cellular FLICE-inhibitory protein splice variants inhibit different steps of caspase- 8 activation at the CD95 death-inducing signaling complex., J. Biol. Chem. 276 (2001) 20633-40.

[61] D.W. Chang, Z. Xing, Y. Pan, A. Algeciras-Schimnich, B.C. Barnhart, S. Yaish-Ohad, et al., cFLIP(L) is a dual function regulator for caspase-8 activation and CD95-mediated apoptosis., EMBO J. 21 (2002) 3704-14.

[62] J. Syken, T. De-Medina, K. Münger, TID1, a human homolog of the Drosophila tumor suppressor 1(2)tid, encodes two mitochondrial modulators of apoptosis with opposing functions., Proc. Natl. Acad. Sci. U. S. A. 96 (1999) 8499-504.

[63] J.R. Leeman, T.D. Gilmore, Alternative splicing in the $\{$ NF-kappaB $\}$ signaling pathway, Gene. 423 (2008) 97-107.

[64] Y.-R. Cho, L. Shi, M. Ramanathan, A. Zhang, A probabilistic framework to predict protein function from interaction data integrated with semantic knowledge., BMC Bioinformatics. 9 (2008) 382. doi:10.1186/1471-2105-9-382.

[65] M.D. McDowall, M.S. Scott, G.J. Barton, PIPs: human protein-protein interaction prediction database., Nucleic Acids Res. 37 (2009) D651-6. doi:10.1093/nar/gkn870. 
[66] D.R. Rhodes, S.A. Tomlins, S. Varambally, V. Mahavisno, T. Barrette, S. Kalyana-Sundaram, et al., Probabilistic model of the human protein-protein interaction network., Nat. Biotechnol. 23 (2005) 951-9. doi:10.1038/nbt1103.

[67] M.S. Scott, G.J. Barton, Probabilistic prediction and ranking of human protein-protein interactions., BMC Bioinformatics. 8 (2007) 239. doi:10.1186/1471-2105-8-239.

[68] D. Szklarczyk, A. Franceschini, M. Kuhn, M. Simonovic, A. Roth, P. Minguez, et al., The STRING database in 2011: functional interaction networks of proteins, globally integrated and scored., Nucleic Acids Res. 39 (2011) D561-8. doi:10.1093/nar/gkq973.

[69] K.R. Brown, I. Jurisica, Online predicted human interaction database., Bioinformatics. 21 (2005) 2076-82. doi:10.1093/bioinformatics/bti273.

[70] T.-W. Huang, A.-C. Tien, W.-S. Huang, Y.-C.G. Lee, C.-L. Peng, H.-H. Tseng, et al., POINT: a database for the prediction of protein-protein interactions based on the orthologous interactome., Bioinformatics. 20 (2004) 3273-6. doi:10.1093/bioinformatics/bth366.

[71] S. Kerrien, B. Aranda, L. Breuza, A. Bridge, F. Broackes-Carter, C. Chen, et al., The IntAct molecular interaction database in 2012., Nucleic Acids Res. 40 (2012) D841-6.

[72] S. Yellaboina, A. Tasneem, D. V Zaykin, B. Raghavachari, R. Jothi, DOMINE: a comprehensive collection of known and predicted domain-domain interactions., Nucleic Acids Res. 39 (2011) D730-5. doi:10.1093/nar/gkq1229.

[73] B. Raghavachari, A. Tasneem, T.M. Przytycka, R. Jothi, DOMINE: a database of protein domain interactions., Nucleic Acids Res. 36 (2008) D656-61. doi:10.1093/nar/gkm761.

[74] C.-S. Yu, Y.-C. Chen, C.-H. Lu, J.-K. Hwang, Prediction of protein subcellular localization., Proteins. 64 (2006) 643-51. doi:10.1002/prot.21018.

[75] M. Kanehisa, S. Goto, KEGG: kyoto encyclopedia of genes and genomes., Nucleic Acids Res. 28 (2000) 27-30.

[76] K.S. Vetrivel, G. Thinakaran, Membrane rafts in Alzheimer's disease beta-amyloid production., Biochim. Biophys. Acta. 1801 (2010) 860-7. doi:10.1016/j.bbalip.2010.03.007.

[77] K. Tang, C. Wang, C. Shen, S. Sheng, R. Ravid, N. Jing, Identification of a novel alternative splicing isoform of human amyloid precursor protein gene, APP639., Eur. J. Neurosci. 18 (2003) $102-8$.

[78] S. Djebali, C.A. Davis, A. Merkel, A. Dobin, T. Lassmann, A. Mortazavi, et al., Landscape of transcription in human cells., Nature. 489 (2012) 101-8. 